\title{
Camarón de río munchilla (Macrobrachium carcinus) en cautiverio en la Estación Ambiental de Sangaral Playa del Medio, Guapi
}

\section{Shrimp of river munchilla (Macrobrachium carcinus) in captivity in the Environmental Station of Sangaral Playa del Medio, Guapi}

\section{Gil Milena Grueso Romero*}

\section{RESUMEN}

El camarón munchilla ( Macrobrachium carcinus), es una especie acuática de la familia de los crustáceos de gran importancia económica en la costa pacífica. Esta investigación tuvo como finalidad, evaluar dos dietas con productos de la zona para monitorear su ganancia de peso, además conocer otros aspectos como el comportamiento de la especie en cautiverio y aspectos reproductivos, porque un gran número de familias dependen económicamente de esta actividad en el Pacífico caucano. En la investigación participó la comunidad, se sembraron las especies en estanques divididos en dos con las mismas condiciones fisicoquímicas. Finalmente se constató que la ganancia de peso fue mayor en la dieta constituida afrecho de maíz, de coco y comején; los individuos presentaron alto grado de agresividad; las hembras que se encontraban en reproducción eran más susceptibles a ser agredidas; los animales soportan condiciones adversas de temperatura, pero no son tolerantes a aguas turbias, se encuentran en aguas dulces y salobres; el alimento concentrado comercial no es apetecido por ellos y sus principales enemigos son la tortuga tapacula (Kinosterno dunni) y la cangrejo de quebradas.

Palabras clave: Camarón; Dieta.

\section{ABSTRACT}

The shrimp munchilla (Macrobrachium carcinus), is an aquatic species of the family of the crustaceans of great economic importance in the pacific coast. This investigation had as an aim, to evaluate two diets with products of the zone to monitor its gain of weight, in addition to know other reproductive aspects like the behavior the species in captivity and aspects, since a great one I number of families depend economically on this activity in the Pacific Caucano. In the investigation I participate the community, seeded the species in pools divided in two with the same physico-chemical conditions. Finally he was stated that the gain of weight was greater in the constituted diet maize bran, of the Coco and termite, the individuals presented/displayed stop aggressiveness degree, the females that were in reproduction were more susceptible to being attacked, the animal support adverse conditions of temperature, but they are not tolerant to turbid waters, are in fresh waters and brackish, the food commercial concentrate is not desired by them and their main enemies are the turtle tapacula (Kinosterno dunni) and the crab of gorges.

Keywords: Shrimp; Diet.

(C) Instituto de Investigaciones Ambientales del Pacífico «John Von Neumann»
Administradora Agropecuaria, Administradora Técnico Regional, Instituto de Investigaciones Ambientales del Pacífico (IIAP), Guapi, Colombia. e-mail: gilmilena@yahoo.es Recibido: marzo 2, 2009 Aceptado: marzo 16, 2009 


\section{Bioetnia Volumen 6 № 1 (enero-junio), 2009}

\section{INTRODUCCIÓN}

El camarón munchilla Macrobrachium carcinus, es una especie acuática de la familia de los crustáceos de gran importancia económica en esta zona del país, debido a su gran demanda comercial por la exquisitez de su carne y a su expectativa como cultivo comercial. El objetivo de esta investigación fue evaluar dos dietas con productos de la zona para conocer la ganancia de peso de los animales en cada una de las dietas, además de otros aspectos como el comportamiento de la especie en cautiverio, aspectos reproductivos porque un gran número de familias dependen económicamente de esta actividad extractiva y es tradicional que sea realizada en exclusiva por las mujeres del Pacífico caucano.Existe literatura de estudios que se han realizado sobre la taxonomía y biología del género Macrobrachium. En Suramérica, especialmente en Colombia se han adelantados varios, entre otros, Granados (1984) estudió la biología y aspectos poblacionales del langostino del río Macrobrachium carcinus en algunas áreas de los estados de Michoacán y Guerrero, México y Guzman y Kensler (1977) realizaron estudios de varias especies del géneroMacrobrachium y sobre sus posibilidades de cultivo en la cuenca baja del río Balsas, Michoacán, Guerrero, México.

Los camarones del géneroMacrobrachium están clasificados en la familia Palaemonidae, orden Decápoda, clase Crustáceo y comprenden un grupo de más de 100 especies (New y Singholka, 1984). Algunas de estas especies tienen valor económico desde el punto de vista pesquero y han venido siendo usadas desde tiempos remotos para el consumo humano en muchos países, incluyendo Colombia. Aunque el cultivo de Macrobrachium conforma apenas $5 \%$ de la producción camaronera mundial, tiene una importancia considerable en el sureste asiático, siendo mucho menor en Latinoamérica (New, 1990).

La especie Macrobrachium carcinus, cuya biología básica es desconocida en gran parte y que no es cultivada a gran escala por su agresividad, lo convierte en una especie difícil de manejar. Verdad a medias, porque nadie ha estudiado la naturaleza de esa agresividad, que en realidad ocurre marcadamente entre individuos del mismo sexo ni ha tratado de eliminarla por medio de manipulaciones genéticas. Por otra parte, este camarón es una especie ruda y más resistente que otros Macrobrachium, tolerando condiciones ambientales adversas y casi cualquier clase de manejo. Finalmente, por su dieta omnívora, acepta todo tipo de alimento, dando ocasión para aprovechar restos de comida o de utilizar insumos locales como la yuca o la pulpa de coco (Mago, 1995).

Durante el estudio se constató que la ganancia de peso fue mayor en la dieta constituida por afrecho de maíz, de coco y comején (animal pequeño). Los individuos presentaron alto grado de agresividad viéndose este comportamiento refleja- do en casos de canibalismo aun teniendo suficiente comida; las hembras que se encontraban en reproducción eran más susceptibles a ser agredidas, los animales soportan condiciones adversas de temperatura, pero no son tolerantes a aguas turbias, se encuentran en aguas dulces y salobres; el alimento concentrado comercial no es apetecido por ellos, sus principales enemigos son la tortuga tapacula (Kinosterno dunni) y la cangrejo de quebradas.

\section{ÁREA DE ESTUDIO}

La Estación Ambiental de Sangaral, Playa del Medio se localiza en el municipio de Guapi en el departamento del Cauca. Guapi se encuentra ubicado al suroccidente del departamento, bordeando la vertiente del Pacífico colombiano, a orillas del río Guapi, sobre una altura de 5 msnmy una temperatura promedio de $29^{\circ} \mathrm{C}$. Tiene una superficie de $2.688 \mathrm{~km}^{2}$; según el Anuario Estadístico del DANE limita por el norte con el océano Pacífico y el municipio de Timbiquí, por el este con el municipio de Timbiquí y Argelia, al oeste con el océano Pacífico y por el sur con el municipio de Santa Bárbara de Iscuandé, Nariño. Su ubicación geográfica es de 2³3'23" de latitud norte y $77^{\circ} 51^{\prime} 50^{\prime \prime}$ de longitud oeste y se encuentra distante $600 \mathrm{~km}$ de Popayán.

El municipio de Guapi está constituido esencialmente por población afrocolombiana que representa más de1 $97 \%$ de los habitantes distribuidos a lo largo y ancho de la región con sus respectivas variaciones dialectales y de costumbres, y el 3\% restante está distribuido entre indígenas y mestizos. Según el Censo del Departamento Administrativo Nacional de Estadísticas (DANE) de 1993, Guapi presentaba una población total de 23.505 habitantes, en el censo del año 2005 la población subió a un total de 28.663, para un aumento de 5.158 habitantes. Estas poblaciones rurales se encuentran organizadas en consejos comunitarios, creados mediante la Ley 70 de 1993 o Ley de Comunidades Negras.

En el caso de la zona rural, se precisa que existen 25 corregimientos y 30 veredas distribuidas a lo largo de 5 ríos principales. Las actividades económicas que conforman la base productiva del municipio, características de la región del Pacífico, están relacionadas principalmente con las del sector primario: agricultura (coco, arroz, naidí chontaduro, papachina, plátano, maíz); pesca (camarón, piangua y pesca blanca), minería y explotación maderera.

En la zona predomina el clima propio de la región Pacífico, altas temperaturas que oscilan alrededor de $\operatorname{los} 28^{\circ} \mathrm{C}$, la humedad relativa fluctúa entre $80 \%$ y $95 \%$ y una precipitación anual entre 4.000 y $6.000 \mathrm{~mm}$ al año. La presencia de ríos navegables y la cercanía al mar, hacen que la economía sea dependiente de los recursos hídricos. Las cuencas más destacadas son las del río Guapi y la del río Guajuí, donde se presentan esteros principales como el Loro, Limones, Quiroga, 


\section{Camarón de munchilla en cautiverio. GM Grueso}

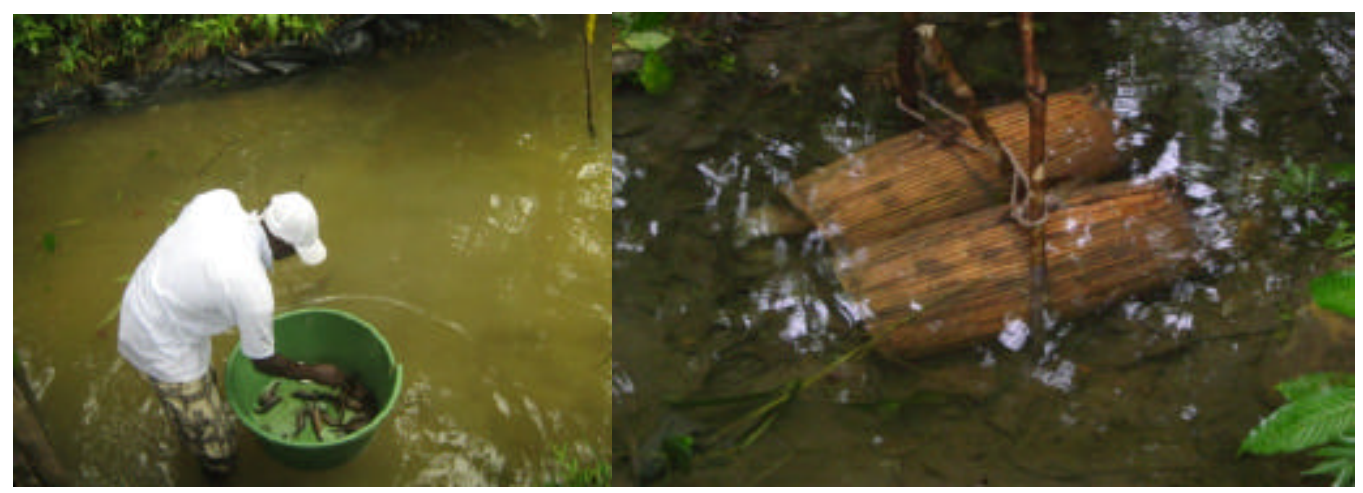

Figura 1. Siembra y captura de camarones para muestreo

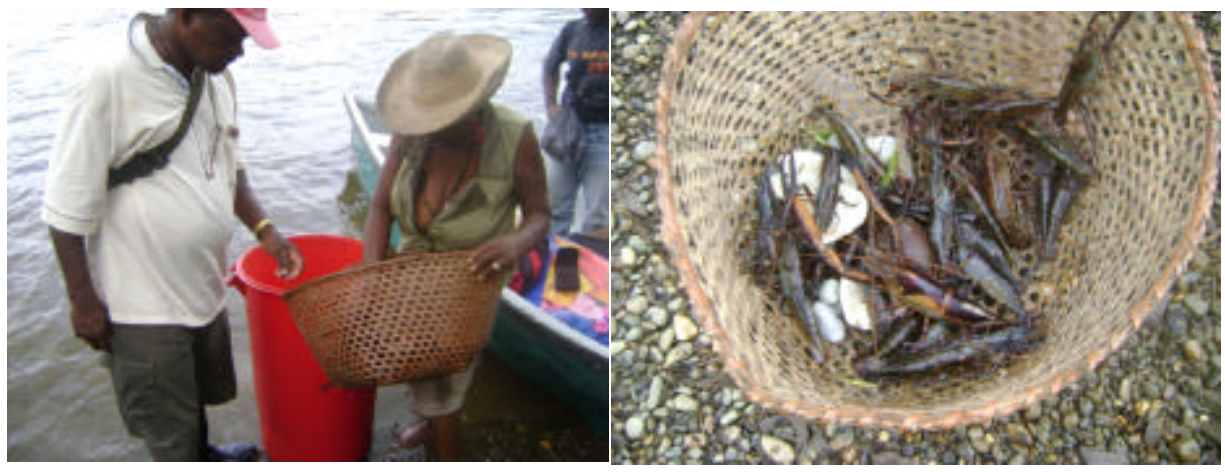

Figura 2. Compra de camarones y muestreo

Playa Blanca, Los Obregones o Pejesapo, el Barrero (canal) y Playa del Medio donde se localiza la estación Ambiental de Sangaral (Alcaldia Municipal de Guapi, 2008).

\section{MÉTODOS}

La metodología de investigación fue acción-participación y se involucró en el desarrollo la investigación a miembros de la comunidad del área de influencia, a mujeres que tradicionalmente realizan la actividad y a estudiantes. Para dar inicio a esta investigación la primera actividad que se realizó fue la adecuación de los estanques, que consistió básicamente en limpieza de fondo y paredes, luego se procedió desinfectarlos con cal y 8 días después se hizo el abonamiento con materia orgánica para incrementar el fitoplancton y zooplancton que servirían de alimento para los individuos; se protegió el lago enmallándolo para evitar la caída de hojas al agua que puedan llegar a alterar las condiciones fisicoquímicas de esta, además se recubrió las paredes del lago con plástico para impedir que los individuos se salgan de él.

Los animales objeto de estudio se recolectaron en la veredas pampa y playa del medio), fueron capturados en catangones con un solo hijo; su captura se hizo instalando catangones en la orilla del río entre las 5 y 6 de la tarde, debido a que los animales tiene hábitos nocturnos (Figura 1); al día siguiente se revisan los catangones y se sacan los individuos capturados.

El estanque se dividió en dos espacios con las mismas condiciones fisicoquímicas, la temperatura promedio fue de $24^{\circ} \mathrm{C}, \mathrm{pH}$ neutro 7 , cada lago tiene un área de $15 \mathrm{~m}^{2}$. Se manejó una densidad de siembra de 3.3 animales por $\mathrm{m}^{2}$. En cada lago se sembraron 50 individuos, para un total de 100 ( 75 hembras y 25 machos), con una relación de 3 hembras por cada macho.

El transporte de los animales se hizo en canastos, sin agua, tapados con costales tratando que no les entrara aire y así evitar su muerte, además durante el proceso de transporte se sumergían los canastos en donde se transportaban los animales cada 30 minutos al agua para oxigenarlos, por recomendación de las mujeres que se dedican a la captura y comercialización de esta especie (Juana Solís -la Pampa camaronera) (Figura 2).

Antes de depositar los animales en el lago se midieron y se pesaron, teniendo un peso inicial promedio de $15.6 \mathrm{~g}$ y una longitud promedio de $8 \mathrm{~cm}$, la temperatura se tomaba a diario con un termómetro de piscina (Figura 3).

Inicialmente se les suministraba en alimento $10 \%$ de la biomasa poblacional. Debido a que se observó agresividad entre ellos se aumentó la cantidad de alimento suministrado a $300 \mathrm{~g}$ diario por lago. Como el hábito de los animales es nocturno, se suministró el alimento en horas de la tarde 5:30 


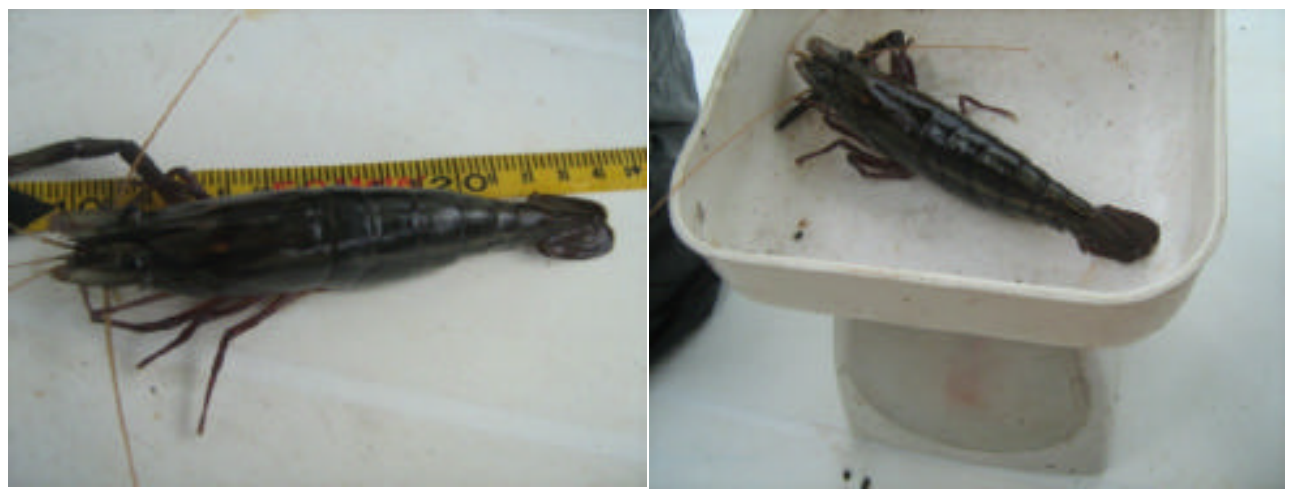

Figura 3. Control de talla y control de peso

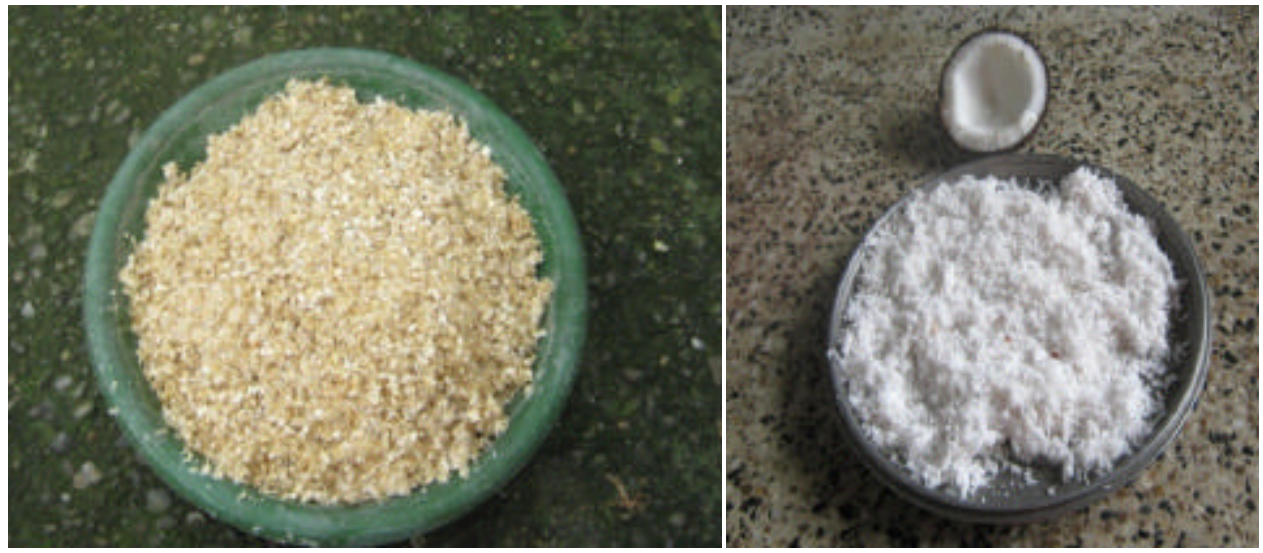

Figura 4. Afrecho de maíz y de coco

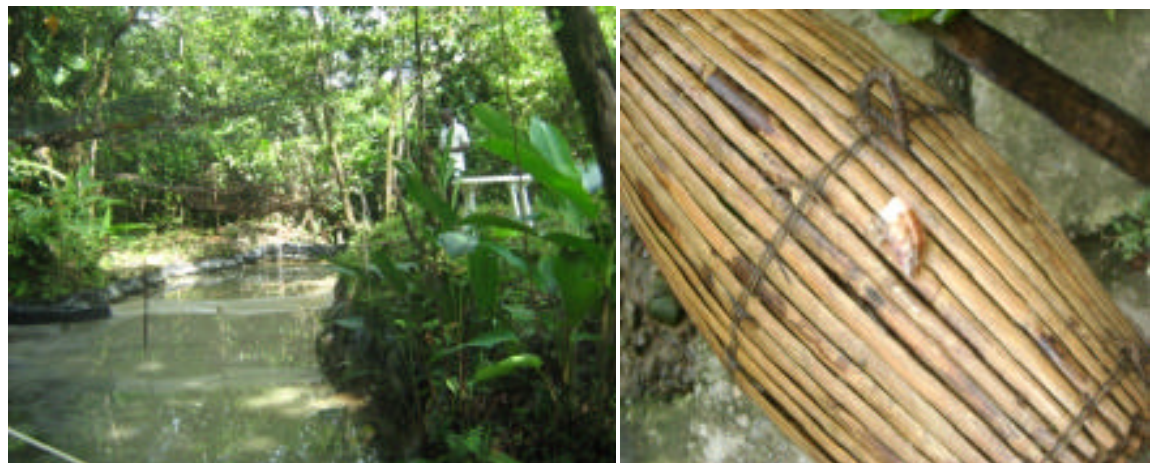

Figura 5. Lago adecuado y catangas (implemento para capturar camarones)

pm en una sola ración; quincenalmente se tomaban datos de peso y talla, la temperatura del lago se tomaba a diario, la entrada del agua era constante abundando más en los períodos de lluvia.

Es importante anotar que inicialmente se inició suministrando concentrado comercial al lago número 2 , pero se observó que los individuos no lo consumían; a los 15 días se cambia la dieta del concentrado comercial por una constituida por productos locales como afrecho de coco, de maíz y sobras de cocina (Figura 4).
A los 15 días de haber iniciado el desarrollo de la investigación, surgió la necesidad de reubicar a los animales en una quebrada cercana que presentara las condiciones ideales para su desarrollo, como mecanismo de disminución de la agresividad y la mortalidad de los individuos (Figura 5).

El procedimiento en general incluyó las siguientes actividades:

- Limpieza de la quebrada

- Se tomaron 15 metros de la quebrada, donde se hizo trincho con maya en la parte de arriba y abajo, este espacio 
se dividió en dos partes iguales, se numeraron los espacios como lago 1 y lago 2.

- Se trasladaron los animales como estaban en el estanque inicial.

Revisión constante de estanques.

- Los individuos se liberaron en una quebrada que pasa por la estación ambiental Sangaral playa del medio, con la participación de miembros de las comunidades vecinas, se liberó un total de 65 individuos, 45 hembras y 20 machos.

\section{RESULTADOS}

Los individuos estudiados provienen de agua dulce y salobre, se utilizaron 100 de ellos, correspondiente a la especie Macrobrachium carcinus.

Diferenciación sexual. Existe una marcada diferenciación sexual lo que hace diferenciar el macho de la hembra; ésta se hace más notable cuando están adultos.

- El macho es más grande que la hembra.

- Las manos, pinzas o tenazas son más grades y carnudas en el macho.

- La abertura sexual en el macho se encuentra en la base del quinto par de patas, mientras que en el hembra se encuentra el la base de tercer par de patas.

- Las hembras presentan una cámara espaciosa incubadora, como la llama Ling (1969), formada por la prolongación de las pleuras abdominales, en donde mantienen los huevos al momento de la reproducción.

Madurez sexual. Debido al tiempo de investigación, no se pudo establecer con exactitud a qué edad los individuos alcanzan su madurez sexual; lo que sí se pudo corroborar es que cuando alcanzan un talla que oscila entre 12 y $15 \mathrm{~cm}$, se presenta dicho estado. Es de anotar que cuando las hembras se encuentran en madurez sexual presentan un color verde amarillento intenso y pese a que durante el estudio lo alcanzaron (hembras enhuevadas), no se pudo contabilizar el número de larvas debido a que el ojo de la maya que delimitaba la quebrada permitió que salieran del área de estudio (hembras enhuevadas).

Fecundidad. La cantidad de huevos esta relacionada con el peso y el tamaño del individuo; mientras más pesen las hembras y su talla sea más grande asimismo es su capacidad de producción y almacenamiento de los huevos en su abdomen.

Porcentaje de mortalidad. El porcentaje de mortalidad fue $35 \%$, considerado muy alto; se cree que esto obedece a la alta agresividad. La mortalidad en las hembras fue mayor que en los machos; se observó que en el período de muda y cuando éstas estaban en período de reproducción se presentaba mayor agresividad hacia ellas, lo que puede suceder como resultado de la ingesta de huevos.

El porcentaje de mortalidad para ellago 1 fue $10 \%$. En el lago
2 la mortalidad fue mayor porque no aceptaron el concentrado comercial que se les suministraba inicialmente, de ahí que se presentara el canibalismo, lo que incrementó la mortalidad hasta en $25 \%$.

Alimentación. Los alimentos suministraron durante las horas de la tarde, debido a los hábitos nocturnos de los individuos; se inició suministrando $10 \%$ de la biomasa, 78 gramos de alimento diario por lago y luego se les aumentó la cantidad de alimento a 300 gramos para disminuir el alto grado de canibalismo que se estaba presentando. Se suministraron en total 49.920 gramos de alimento en 180 días. La dieta 1 consistió afrecho de maíz, afrecho de coco, comején; la dieta 2 en sobras de cocina, maduro y afrecho de maíz. El peso y la biomasa inicial fue igual para ambos lagos; el peso fue de 15.6 gramos promedio y la biomasa de 780 gramos.

Dieta número 1 (lago 1). Al momento de la liberación los individuos tenían un peso de 20.90 gramos promedio, es decir habían ganado 5.3 gramos en 6 meses, que significa una ganancia de 0.029 gramos diario y la biomasa promedio hasta el momento es de 954 gramos.

Dieta número 2 (lago 2). Durante la liberación los individuos tenían un peso 19.07 gramos promedio es decir, ganaron 3.47 gramos en 6 meses, lo que implica una ganancia de 0.019 gramos diario y una biomasa de 740 gramos.

Talla. La talla promedio inicial fue de $8 \mathrm{~cm}$ para ambos lagos.

Talla, lago 1. La talla de liberación fue de $14 \mathrm{~cm}$ en promedio, obteniendo una ganancia de $6 \mathrm{~cm}$ durante la investigación.

Talla lago 2. La talla de liberación fue de $12.8 \mathrm{~cm}$ en promedio, obteniendo una ganancia de $4.8 \mathrm{~cm}$ durante la investigación.

Parámetros fisicoquímicos. Las variables ambientales juegan un papel muy importante en el desarrollo de los individuos, porque determinan muchos de sus comportamientos. Se manejó un $\mathrm{pH}$ neutro y una temperatura promedio de $24^{\circ} \mathrm{C}$ en los lagos o estanques; la temperatura en la quebrada fue $20^{\circ} \mathrm{C}$.

Muestreo y toma de datos. El monitoreo de los animales se realizó a diario en horas de la tarde siendo este el momento de observar los animales con mayor facilidad. Se aprovechó este tiempo para retirar los animales muertos o que habían sido altamente agredidos; cada 15 días se tomaron datos de peso y talla; para capturar a los animales se introducían catangas en las noches y en las mañanas con el fin de tomar datos de talla y peso al $10 \%$ de la población.

Comportamiento general de la especie. Se encontró que los individuos de Macrobrachium carcinus son animales acuáticos, que viven y se desarrollan en aguas dulces o salobres de las zonas tropicales. Durante el día se mantienen encuevados entre las piedras o raíces sumergidas de los árboles, en agujeros excavados en el lodo o en lugares prote- 


\section{Bioetnia Volumen 6 № 1 (enero-junio), 2009}

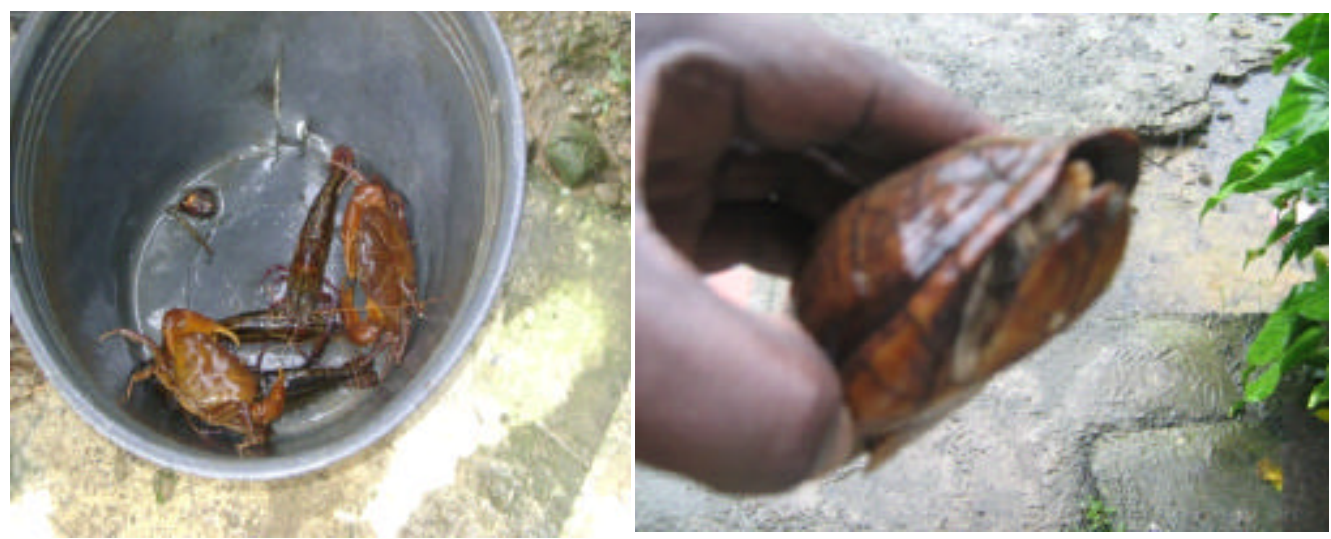

Figura 6. Cangrejode agua dulce y tortuga tapacula (Kinosternom dunni)

gidos como las pozas. Son nocturnos, por eso en las noches salen a buscar su alimento. En general es un animal que aparenta un comportamiento tranquilo, pero son muy territoriales y agresivos; presentan conducta de escape y canibalismo entre ellos, sobre todo cuando no existe comida disponible suficiente. Siempre se encuentran a la expectativa y se defienden con sus tenazas, moviéndose hacia atrás.

Comportamiento en cautiverio. Se observó que este tipo de camarones no se adaptan a las condiciones de estanques con aguas tranquilas o estancadas, prefieren aguas cristalinas y que presenten movimiento constante (corrientosas). Por otro, lado se pudo observar que aumenta la agresividad cuando el agua está turbia porque se sienten atacados.

Taxonomía de la especie Phylum arthropoda

Género: Macrobrachium

Clase: Crustáceo

Familia: Palaemonidae

Orden: Decápoda.

\section{DISCUSIÓN}

En los datos registrados en la talla y el peso de los individuos se aprecia una diferencia entre estas dos variables entre los animales del lago 1 y del lago 2, obteniendo mayor ganancia (5.3 g) en lago 1, cuya dieta estaba conformada por afrecho de maíz, coco y comején, mientras que la ganancia de peso para el lago número 2 fue de 3.47 gramos; igualmente mientras que los individuos del lago 1 ganaron $6 \mathrm{~cm}$ los del lago 2 sólo ganaron $4.8 \mathrm{~cm}$. Un dato curioso fue que los animales no aceptaron el concentrado comercial, lo que generó la necesidad de sustituirlo por una dieta conformada por productos de la zona.

Por otro lado el porcentaje de mortalidad en general fue alto $(35 \%)$, siendo mayor cuando los animales estaban en estanques de aguas tranquilas y disminuyendo considerablemente cuando se trasladaron a la quebrada que presentaba condiciones propias de su estado natural. Las hembras pre- sentaron mayor índice de mortalidad debido a que eran más susceptibles a ser atacadas cuando estaban enhuevadas (reproducción).

Es pertinente anotar que se presentó mayor mortalidad en el lago 2, lo que pudo obedecer a la no aceptación del concentrado comercial inicialmente, produciendo que se presentara mayor grado de canibalismo e incrementando la mortalidad al $25 \%$.

Se logró corroborar lo expuesto por Valdés y Gozales (2001), acerca de que los principales enemigos del camarón monchilla, la tortuga tapacula (Kinosterno dunni) y el cangrejo de quebradas, ya que se determinó la presencia de especies similares en la zona que pudieron influir en los índices de mortalidad obtenidos (Figura 6). Este tipo de camarones mostró además que aunque soportan condiciones adversas de temperatura, no son tolerantes a aguas turbias, se encuentran mejor en aguas dulces y salobres y el alimento concentrado comercial no es apetecido por ellos, situaciones que se pudieron comprobar en campo y que permiten inferir sobre el comportamiento de los mismos y la toma de decisiones adecuadas para su cultivo en beneficio de la comunidad.

\section{CONCLUSIONES}

El camarón munchilla (Macrobrachium carcinus) presenta mayor agresividad en estanques de aguas tranquilas y que no tienen sitos donde resguardase como materiales rocosos o troncos de palos huecos, lo que influye de manera directa en el aumento de la mortalidad; los individuos presentan comportamiento poco agresivo en su estado natural en aguas corrientosas y cristalinas, son animales territoriales de hábitos nocturnos, omnívoros que prefieren alimento de subproductos de fácil consecución en la zona como afrechos de coco y de maíz, comején y sobras de cocina, entre otros, lo que hace que su cultivo sea económico.

Las hembras son más vulnerables de ataque cuando están en período de reproducción, hay que tener en cuenta que la 
cría se debe hacer en su hábitat natural como quebradas y riachuelos, donde preferiblemente se suministra el alimento en las noches porque tienen un hábito nocturno.

Es importante tener en cuenta en la conformación de las dietas, que estas contengan proteína animal (comején, lombriz entre otros), para contribuir en aumentar su ganancia de peso.

\section{RECOMENDACIONES}

- Se recomienda que para el cultivo del camarón munchilla el fondo del estanque debe ser de piedra, peña o balastro con troncos huecos que permita el resguardo de los individuados, con agua cristalina y libre de sustancias tóxicas.

- Es importante manejar baja densidad de siembra para disminuir las posibilidades de comportamientos de canibalismo.

- En lo posible separar las hembras de los machos cuando están en período de reproducción porque son susceptible a ser atacadas.

- Se debe tener en cuenta al momento de la compra de los camarones que tengan las dos tenazas, debido a que son los órganos de defensa y herramientas fundamentales para la alimentación.

\section{LITERATURA CITADA}

Alcaldía Municipal de Guapi. 2008. Plan de Desarrollo Municipal de Guapi Cauca 2008-2011. Trabajando con experiencia. Guapi: Alcaldía Municipal de Guapi.

Granados, A. A., 1984. Aspectos reproductivos del «camarón prieto» Macrobrachium acanthurus (Wiegmann, 1836) en la cuenca del río González, Tabasco, México (Crustacea: Decapoda; Palaemonidae). An Inst Cien Mar Limnol. 11: 1-22.

Guzmán, M, C. Kensler. 1977. Informe del proyecto biología, ecología y pesquería de los langostinos del género Macrobrachium en México. Posibilidades de cultivo del langostino Macrobrachium, en el área de la ciudad de Lázaro Cárdenas. Mich. y zonas de influencia. Centro Cienc del Mar y Limnol UNAM. México.19 p. (Mimeogr.)

López A.C., P.C. Sierra-Correa, J.C Rodríguez, J.L. Freyre-Palua (eds.). 2003. Plan de manejo integrado de la zona costera del complejo de las bocanas Guapi Iscuandé, Pacífico colombiano. Serie de Documentos Generales INVEMAR $N^{\circ}$ 17. Fase II. INVEMAR-CRC CORPONARIÑO-IIAP. Santa Marta: Ministerio de Ambiente, Vivienda y Desarrollo Territorial; $138 \mathrm{p}+6$ anexos

Mago, F. 1995. El cultivo del camarón de río Macrobrachium carcinus, un potencial desestimado en Venezuela. Maracay: FONAIAP Centro de Investigaciones Agropecuarias del Estado Anzoátegui. Estación Local Barcelona.

New, M., S. Singholka. 1984. Manual para el cultivo de camarónMacrobrachium rosenbergii. Fisheries Technical Paper $\mathrm{N}^{\circ} 225$. Roma: FAO;118 p

New, M. 1990. Freshwater prawn cultura: a review. Aquaculture. 88: 99-143.

Valdes, M., S. Gonzáles, 2001. Tortuga Lora. Escuela Juana de Asbaje y Ramírez. Tamaulipas, México. (on line). http://www.semarnat.gob.mx/ especies/tortuga/index.shtml 